\title{
Estructura profunda del Zagros y de la meseta de Irán: modelo geofísico y petrológico
}

\author{
L. TUNINI, I. JIMÉNEZ-MUNT \& M. FERNÁNDEZ \\ Instituto de Ciencias de la Tierra Jaume Almera, CSIC, Sole Sabarís s/n, 08028 Barcelona, España. \\ e-mail: 1tunini@ictja.csic.es
}

Recibido: $29 / 07 / 2011$

Aceptado: 20/09/2011

\begin{abstract}
Resumen
El Zagros, la cadena montañosa que atraviesa todo Irán desde NO hacia SE, es el resultado de la colisión de la placa de Arabia con la placa Euroasiática durante el Cenozoico. Una anomalía de bajas velocidades sísmicas (Maggi \& Priestley, 2005; Alinaghi et al., 2007) y el ajuste conjunto de elevación, campos potenciales y datos térmicos (Molinaro et al., 2005), revelan un adelgazamiento del manto superior debajo del orógeno.

Utilizando un método de modelización numérica que integra datos de elevación, geoide, gravedad, flujo de calor superficial, sísmica y petrofísica (Afonso et al., 2008), se investiga la estructura del manto hasta los $400 \mathrm{~km}$ de profundidad a lo largo de un perfil que atraviesa el sector más occidental del Zagros, pasa por el Alborz y alcanza la costa del Mar Caspio.

El resultado muestra un considerable déficit de masa en el manto litosférico debajo del Zagros, que resolvemos con variaciones del espesor del manto litosférico. La base de la litosfera, que debajo del Foreland Basin roza los $230 \mathrm{~km}$ de profundidad, se levanta hacia los $\sim 120 \mathrm{~km}$ debajo de la Sinandaj Sirjan Zone. El adelgazamiento comprende toda la zona debajo del Zagros y del Alborz, y está acompañado por una anomalía negativa de densidad y una temperatura más elevada. Para obtener un ajuste óptimo de todos los datos, ha sido necesario utilizar tres diferentes composiciones mantélicas: un Proton-6 (Griffin et al., 2008) para el manto debajo de la cuenca Mesopotámica; un Tecton Tc-2 (Griffin et al., 2008) para el manto adelgazado debajo del orógeno, y una composición diferente, procedente de la composición de las ofiolitas de Sahneh, para el manto debajo de la placa Eurasiática.

Palabras clave: manto litosférico, composición mantélica, LAB.
\end{abstract}

\section{Deep structure across the Zagros Mountains and the Iranian Plateau. An integrated geophysical and petrological approach}

\begin{abstract}
The Zagros, mountains which cross Iran from NW to SE, were formed by the collision between the Arabian and Eurasian plates during the Cenozoic. A low seismic velocities anomaly (Maggi \& Priestley, 2005; Alinaghi et al., 2007) and the integrated fitting of elevation, potential fields and thermal data (Molinaro et al., 2005), suggest a thinning in the upper mantle below the orogen.

Using a method (Afonso et al., 2008) which combines elevation, geoid, gravity, superficial heat flow, seismic and petrological data, we investigate the mantle structure down to $400 \mathrm{~km}$ depth of a profile which crosses the westernmost part of the Zagros Mountains, passes the Alborz and reaches the southern coast of the Caspian Sea.

The result shows a significant deficit of mass in the lithospheric mantle beneath the Zagros and we solve that with thickness variation of the lithospheric mantle. The lithosphere-astenosphere boundary (LAB), which is almost $230 \mathrm{~km}$ deep below the Foreland Basin, goes up to $120 \mathrm{~km}$ depth below the Sinandaj Sirjan Zone. A mantle thinning affects the whole area below the Zagros and the Alborz Mountains and is characterized by a negative density anomaly and a higher temperature. It was
\end{abstract}


necessary to use three different mantle compositions to reach the best fit of the data: a Proton-6 (Griffin et al., 2008) for the mantle below the Mesopotamian foreland; a Tecton Tc-2 (Griffin et al., 2008) for the mantle below the orogen, and a different composition derived from Sahneh ophiolite composition for the mantle below the Eurasian plate.

Keywords: lithospheric mantle, mantle composition, LAB.

Sumario: Introducción. 1. Contexto geológico. 2. Datos y método. 2.1 Datos geofísicos. 2.2 La corteza. 2.3 El manto. 3. Resultados y discusión 4. Conclusiones. Agradecimientos. Referencias bibliográficas.

\section{Referencia normalizada}

Tunini, L., Jiménez-Munt, I., Fernández, M., (2011). Estructura profunda del Zagros y de la meseta de Irán: modelo geofísico y petrológico. Física de la Tierra Vol. 23 Núm. 1 (2011): 93-111

\section{Introducción}

Las zonas de colisión continental han sido clásicamente identificadas como áreas con una corteza engrosada y un manto litosférico también engrosado por duplicación o por subducción como resultado de un largo periodo de convergencia tectónica. Sin embargo recientemente se ha propuesto que parte de la elevada topografía en orógenos es soportada por un considerable déficit de masa bajo la corteza atribuido a un adelgazamiento del manto litosférico.

El Zagros (Fig. 1) es una cadena montañosa situada entre la placa Arábiga y la Euroasiática, que atraviesa todo Irán desde $\mathrm{NO}$ a SE, con una altitud media de 2000 $\mathrm{m}$ y una longitud de más de $2000 \mathrm{~km}$. El orógeno es el resultado de la colisión de la placa de Arabia con la placa Euroasiática durante el Cenozoico, que causó también el cierre del Neo-Tetys y su subducción bajo de la placa Euroasiática.

Dehghani \& Makris (1984) publicaron un mapa de anomalía de Bouguer que marca una fuerte anomalía negativa con tendencia NO-SE centrada en la Main Zagros Fault (MZF) y calcularon un mínimo absoluto de $-230 \mathrm{mGal}$. De acuerdo con estos autores, esta anomalía proporciona un espesor cortical de $50-55 \mathrm{~km}$ debajo de la Main Zagros Fault y de $\sim 40 \mathrm{~km}$ en la cuenca Mesopotámica. Un estudio de las anomalías de Bouguer más detallado y centrado en el Zagros, llevó a Snyder \& Barazangi (1986) a obtener valores de espesores corticales de $40 \mathrm{~km}$ debajo de la cuenca mesopotámica a $\sim 60 \mathrm{~km}$ debajo de la MZF. Los mismos autores comentaron también que la carga topográfica del orógeno es insuficiente para explicar las observaciones en términos de simple flexión litosférica.

Hatzfeld et al. (2003) utilizaron el análisis de las funciones receptoras de registros sísmicos para inferir un espesor cortical de $\sim 46 \mathrm{~km}$ debajo de una estación situada en el Zagros central. Trabajos más recientes (Paul et al., 2010) utilizaron la misma técnica usando telesismos para sacar imágenes del Moho. Su perfil norteoccidental (ver ubicación en Fig. 1), muestra una base de la corteza ondulada que aumenta su profundidad hacia el interior de la cadena del Zagros, con una profundidad media del Moho de $42 \pm 2 \mathrm{~km}$ en la cuenca Mesopotámica hasta llegar a un máximo de $56 \pm 2 \mathrm{~km}$ en el lado sur-occidental de la Sanandaj-Sirjan Zone. 


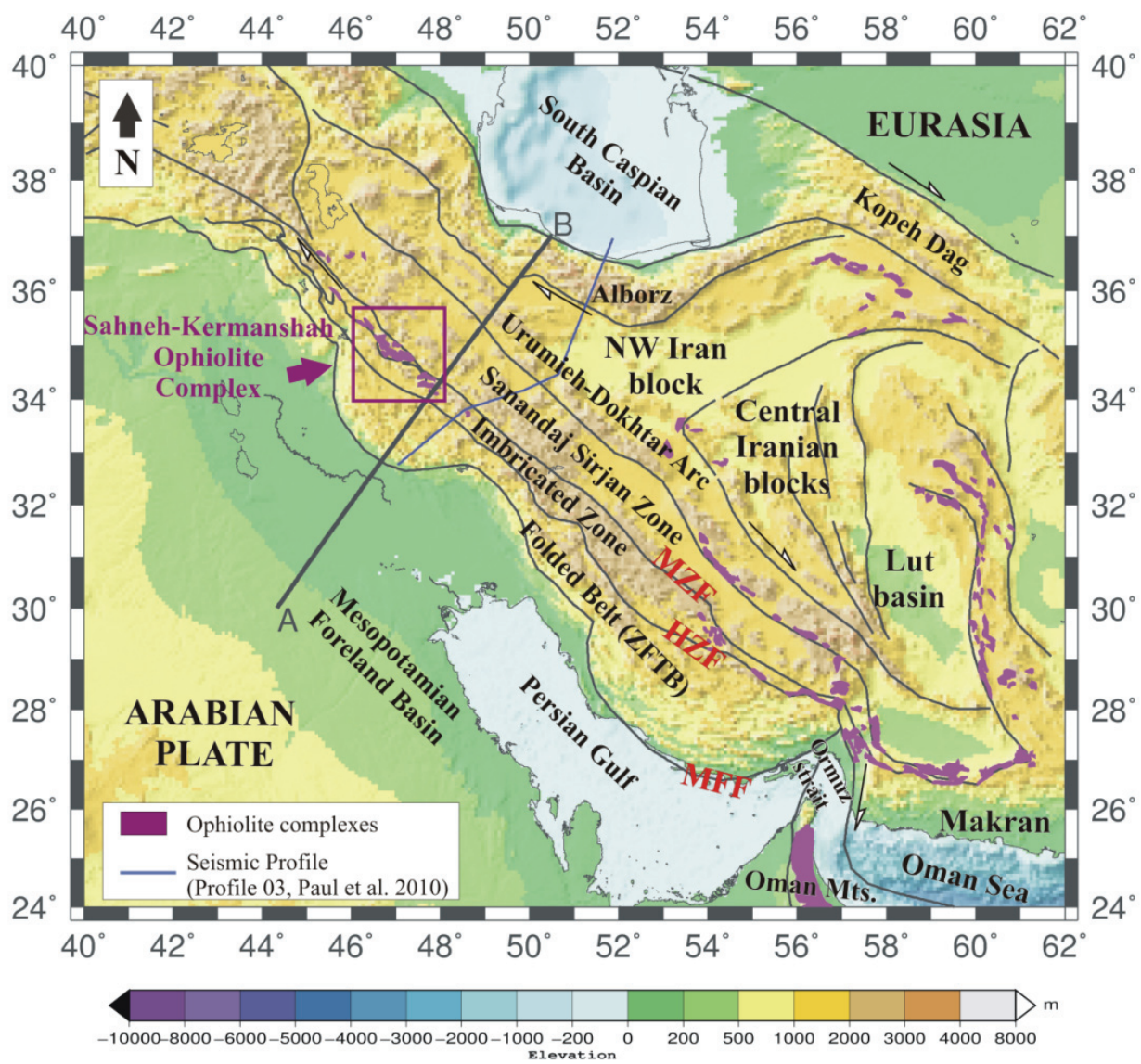

Fig. 1. Contexto tectónico de la zona de colisión Arabia-Eurasia: ubicación del perfil estudiado AB, del complejo ofiolítico de Sahneh-Kermanshah, del perfil sísmico (línea azul) de Paul et al. (2010) y de las principales líneas estructurales de la zona.

Poco se conoce acerca la estructura litosférica del Zagros. La tomografía sísmica, la magnetotelúrica, el volcanismo alcalino y el balance isostático parecen sugerir un adelgazamiento del manto litosférico debajo del orógeno. Una anomalía de bajas velocidades sísmicas (Maggi \& Priestley, 2005; Alinaghi et al., 2007) y el ajuste conjunto de elevación, campos potenciales y datos térmicos (Molinaro et al., 2005), revelan un déficit de masa que parece contradecir la geodinámica clásica de las zonas colisionales. Estos trabajos asumen que la densidad en el manto es función sólo de la temperatura, mientras que la densidad en la astenosfera se mantiene constante.

Sin embargo trabajos recientes (Afonso et al., 2008; Griffin et al. 2008) han puesto de manifiesto que hay otros parámetros que entran en juego para determinar la densidad, como la presión y la composición mineralógica. En el manto las condi- 
ciones de temperatura y presión afectan al equilibrio de las fases minerales y por lo tanto a la composición del mismo. Los trabajos hechos hasta ahora, al no considerar la compresibilidad, los cambios de fases y/o la variabilidad composicional en el manto superior, no pueden ser comparados con tomografías, xenolitos y datos termodinámicos.

En este artículo presentamos un estudio de la estructura litosférica del Zagros empleando una metodología de modelización numérica (Afonso et al., 2008), que tiene en cuenta la petrofísica del manto: su densidad es función no sólo de la temperatura, sino también de la presión y de la composición, y la densidad astenosférica depende de ambas variables (temperatura y presión). Se propone determinar el límite litosfera-astenosfera (LAB) y las características del manto litosférico a lo largo de un perfil SO-NE, situado en la zona norte-occidental de la cadena del Zagros.

El perfil atraviesa perpendicularmente las montañas del Zagros norte-oriental, llegando hasta la costa del Mar Caspio, con una longitud de 980 km (Fig. 1, perfil $\mathrm{AB})$, y que tiene las siguientes coordenadas:

\section{A: $44,33^{\circ}$ Este $-30^{\circ}$ Norte \\ B: $50,5^{\circ}$ Este $-37^{\circ}$ Norte}

La ubicación del perfil en la parte noroeste de Irán, ha sido elegida teniendo en cuenta la mayor disponibilidad de datos posibles, en relación a espesores de sedimentos, valores de profundidad del Moho, estudios sísmicos y cortes geológicos. Para constreñir los espesores corticales se utilizan datos de sísmica, y cortes geológicos. Las composiciones de manto se hallan a partir de datos petrográficos previos o estudios de la evolución composicional de los mantos con composiciones de referencia (Griffin et al. 2008). Así se pretende reducir las incertidumbres y obtener un modelo más robusto del que se podría obtener modelando únicamente una de las variables o combinaciones de estas como comúnmente se hace.

\section{Contexto geológico}

El Zagros es una cadena de pliegues y cabalgamientos sísmicamente activa que se sitúa en el margen norte-oriental de la placa de Arabia, en la costa septentrional del Golfo Pérsico (Fig. 1) y que está generada por la colisión entre la placa Arábiga y la placa Euroasiática, que empezó en el Mioceno y perdura hasta el día de hoy (Alavi, 1994; Agard, 2005; Ghasemi \& Talbot, 2006; Casciello et al., 2009; Homke et al, 2009 y otros).

Las evidencias geológicas indican que el Zagros sufrió varios episodios tectónicos que afectaron a diferentes partes de la cadena (Vergés et al., 2011). Desde el Cámbrico al Plioceno la sedimentación fue tranquila con solo una leve deformación y algún fenómeno de diapirismo salínico en el sector norte-oriental (Imbricated Zone). En el Cretácico superior empezó la orogénesis con el emplazamiento de unidades ofiolíticas procedentes de la corteza oceánica del Neo-Tetys sobre el 
margen norte-oriental de Arabia (Ricou, 1971; Ziegler, 2001). La obducción prosiguió hasta el final del Campanianense, mientras que el resto del Neo-Tetys continuó subduciendo bajo la placa de Irán hasta que empezó la colisión continental en el Oligoceno-Mioceno.

Del Suroeste hacia el Noreste, se atraviesan cinco dominios estructurales en los que tradicionalmente se subdivide el Zagros desde el punto de vista tectónico: 1) la cuenca de Mesopotamia, que representa, junto con el Golfo Pérsico, la cuenca de antepaís que se formó en frente del orógeno. Su espesor cortical se va adelgazando hacia el Suroeste de la plataforma de Arabia; 2) el cinturón Zagros Fold Thrust Belt (ZFTB) o Zagros Folded Belt, caracterizado por amplios pliegues concéntricos y paralelos. Este sistema representa el margen exterior menos deformado del orógeno; 3) la Zona Imbricada (Imbricated Zone) o High Zagros, que es un área compuesta por capas de sedimentos mesozóicos, radiolaritas, restos ofiolíticos, y materiales volcánicos eocénicos, obducidos sobre el margen arábigo (Agard et al., 2005); 4) la Sanandaj Sirjan Zone (SSZ), formada principalmente por rocas metamórficas del Paleozoico al Cretácico, intruídas por plutones calco-alcalinos del Jurásico al Eoceno Inferior (Alavi, 1994). La SSZ representa el corazón metamórfico del proceso colisional en el oeste de Irán. Se caracteriza por ser una zona de fallas inversas que tranportaron, junto a las ofiolitas obducidas, varias capas de unidades estratigráficas, de la zona de sutura en el Noreste hacia el Suroeste, por encima del margen continental pasivo Afro-Arábigo; 5) el arco magmático Urumieh-Dokhtar (UDMA), compuesto por rocas extrusivas e intrusivas toleíticas, calco-alcalinas, y alcalinas enriquecidas en $\mathrm{K}$, con sucesiones volcanoclásticas y piroclásticas asociadas. Las rocas de esta zona son esencialmente mesozóicas. Los materiales volcánicos se deben principalmente a la subducción de la placa de Arabia conteniente restos de la corteza oceánica del Neo-Tetys. El pico de actividad volcánica remonta al Eoceno, y desde el Cuaternario no hay indicio de actividad volcánica (Alavi, 1994). Las ofiolitas presentes en esta zona son datadas $86-81 \mathrm{Ma}$, lo que significa que se emplazaron en el Mesozoico tardío.

Grandes fallas a escala regional marcan los bordes de los dominios estructurales:

1) la Mountain Front Fault (MFF); 2) la High Zagros Fault (HZF); 3) la Main Zagros Fault (MZF) comúnmente aceptada como la zona de sutura entre la placa de Arabia y la placa Euroasiática (Berberian and King, 1981), aunque Alavi (1994) sugirió que la zona de sutura se sitúa entre el Arco Magmático Urumieh Doktar y el Sanandaj Sirjan.

\section{Datos y método}

El algoritmo utilizado (LitMod 2D, Afonso et al., 2008), es un código de elementos finitos que calcula la estructura térmica, el flujo de calor superficial (SHF), anomalías de gravedad, anomalías del geoide, velocidades sísmicas y elevación, a partir de observables globales y de datos composicionales que entran como input en el código. El programa también permite tener un control sobre las variaciones composicionales y térmicas a diferentes profundidades. El método consiste en encontrar una distribución de densidades que mejor ajuste los datos de elevación, flujo de calor, 
anomalías del geoide y gravimetría, así como las velocidades de las ondas sísmicas en el manto.

\subsection{Datos geofísicos}

Las variables geofísicas (elevación, gravedad, geoide, flujo de calor superficial) han sido recopiladas utilizando principalmente bases de datos globales.

La elevación (Fig. 2a) procede del modelo ETOPO1 (Sandwell and Smith, 1997), un modelo de elevación global de la superficie de la Tierra de 1 minuto de arco que integra topografía en tierra y batimetría oceánica, disponible en http://www.ngdc.noaa.gov/mgg/global/global.html. Como se puede observar, la topografía es muy suave al principio del perfil y en correspondencia con la cuenca Mesopotámica, al Norte del Golfo Pérsico. Luego se hace más abrupta y cambia bruscamente del nivel del mar a $\sim 1500 \mathrm{~m}$ en menos de $100 \mathrm{~km}$ en el Zagros Folded Belt, y alcanza cotas de más de $2500 \mathrm{~m}$ en el Zagros central y en el Alborz.

La altura del geoide procede del EGM2008 (Pavlis et al., 2008), un modelo que incorpora datos de anomalías gravimétricas en mallas de $1 \mathrm{x} 1$ minutos, que se extiende hasta los coeficientes de grado y orden 2190. Dado que el geoide refleja la distribución de densidad de toda la Tierra, para utilizarlo como observable en nuestra modelización es necesario quitar las longitudes de onda mayores de $4000 \mathrm{~km}$, es decir, las que son responsables de las anomalías de densidad del manto profundo. Para ello se substraen los armónicos esféricos hasta el grado y orden 8 (Motovalli Anbaran et al., 2010) obteniendo una anomalía del geoide residual, que refleja la distribución de anomalías de densidad más superficiales de los $400 \mathrm{~km}$ aproximadamente (Fig. 2b). E1 EGM2008 muestra, en la zona de estudio, una significativa variación de la altura del geoide, con un mínimo de -16 m localizado en el Golfo Pérsico, y un máximo de $18 \mathrm{~m}$ en el NE, en Anatolia. En general los valores positivos caracterizan el Zagros y el Alborz, mientras una marcada anomalía negativa se observa en el Golfo Pérsico, en la cuenca Mesopotámica, mar Caspio y NE de Kopeh Dag. En la meseta de Irán y en la cuenca de Lut los valores rodean el 0. 

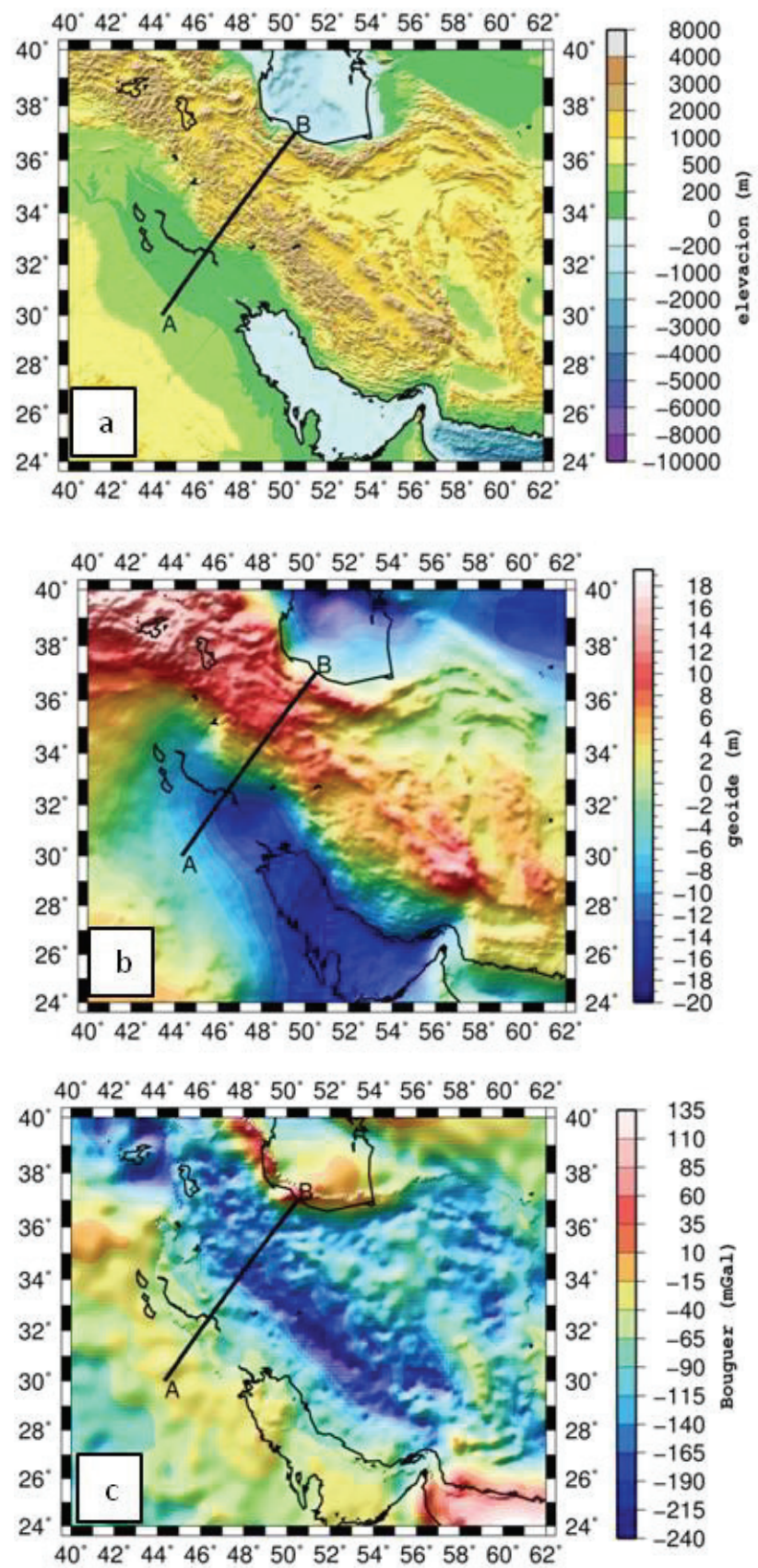

Fig. 2. Observables geofísicos. a) Elevación procedente del modelo ETOPO1 (Sandwell and Smith, 1997). b) Altura del geoide procedente del modelo EGM2008 (Pavlis et al., 2008), donde las longitudes de onda mayores de $4000 \mathrm{~km}$ han sido eliminadas. c) Mapa de anoma- 
lías de Bouguer. En Irán los datos proceden de Getech (grid: $10 \mathrm{~km}$ x $10 \mathrm{~km}$ ). En el resto de la región se ha determinado la anomalía de Bouguer a partir de la anomalía de aire libre (véase explicación en el texto).

Para la anomalía de Bouguer, en el territorio de Irán se han utilizado los datos de Getech, en una malla de $10 \mathrm{~km} \times 10 \mathrm{~km}$ (5 minutos de arco aproximadamente). Para el resto de la región, las anomalías de Bouguer (Fig. 2c) han sido calculadas a partir de la anomalía de aire libre (Sandwell and Smith, 1997), a la cual se aplica la corrección topográfica 3D siguiendo el método ilustrado por Fullea et al. (2008), usando una densidad de reducción de $2670 \mathrm{~kg} / \mathrm{m}^{3}$. La anomalía de Bouguer a lo largo de la cadena del Zagros muestra un mínimo de $-220 \mathrm{mGal}$, que corre paralelamente al orógeno, indicando la presencia de una raíz cortical. En la depresión central de Irán y en la cuenca de Lut (véase su ubicación en Fig. 1), el campo de gravedad alcanza valores de $-80 \mathrm{mGal}$ y $-60 \mathrm{mGal}$ respectivamente, sugiriendo una corteza más adelgazada, mientras que en el Alborz aumenta llegando a los 25 mGal.

\subsection{La corteza}

Nasrabadi et al. (2008) utiliza las estaciones sísmicas de banda ancha de la red INSN (Iranian National Seismic Network) aplicando un método que combina funciones receptoras y dispersión de la velocidad de grupo de las ondas superficiales. Sus resultados indican un engrosamiento de la corteza de hasta $55 \mathrm{~km}$ de profundidad en la Sanandaj Sirjan Zone y en el Alborz (52-53 km de profundidad del Moho), mientras varía entre 38-40 km bajo la meseta de Irán, entre el Zagros y el Alborz. Más recientemente Radjaee et al. (2010), obtiene valores parecidos de espesor cortical: la base de la corteza pasa de $\sim 48 \mathrm{~km}$ debajo de la parte norte de la meseta de Irán a 55-58 km debajo del Alborz central, para luego disminuir a 46 $\mathrm{km}$ en la costa del Mar Caspio.

Un modelo cortical inicial (Fig. 3) ha sido construido a partir de la recopilación de los estudios sísmicos y geológicos (NIOC, National Iranian Oil Company) previos disponibles en la zona. La geometría de los diferentes cuerpos ha sido simplificada acorde a la escala regional a la que trabajamos, y puede llegar a tener un grado de subjetividad bastante elevado considerando que los cortes geológicos no superan los $5 \mathrm{~km}$ de profundidad y no existen sondeos o investigaciones directas profundas en la zona. Sin embargo, las principales líneas de discontinuidad han constituido puntos de referencia clave para la modelización de la corteza. 


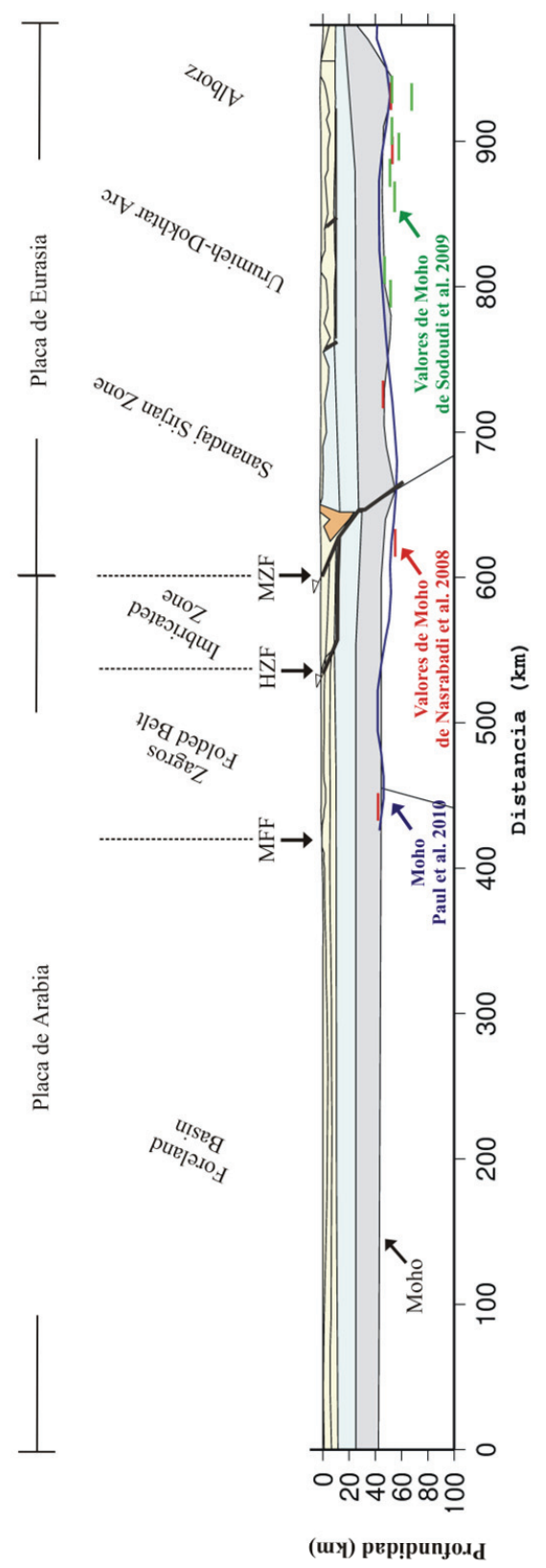

Fig. 3. Modelo cortical a escala del perfil estudiado. La línea azul representa el perfil Zagros03 de Paul et al. (2010) (véase su ubicación en la Fig. 1) obtenido por funciones receptoras, mientras que los segmentos rojos y verdes son los valores de profundidad del Moho de Nasrabadi et al. (2008) y Sodoudi et al. (2009) respectivamente, proyectados sobre el perfil. 
En línea general, a nivel de corteza se observa un engrosamiento a norte de la MZF, debajo de la Sanandaj Sirjan Zone y del arco Urumieh Dokhtar, así como debajo del Alborz. Se puede observar que el modelo respeta los espesores corticales de otros autores (Nasrabadi et al., 2008; Paul et al., 2010) en la Zagros Folded Belt, con valores de $\sim 42 \mathrm{~km}$. Lo mismo vale para el sector del arco magmático y del Alborz (Nasrabadi et al., 2008; Sodoudi et al., 2009), con valores de $43 \mathrm{~km}$ y de $\sim 51 \mathrm{~km}$ respectivamente. En la Imbricated Zone, Paul et al. (2010) detecta un engrosamiento cortical más pronunciado que en nuestro modelo, pero en la Sinandaj Sirjan Zone el modelo concuerda con los valores de Nasrabadi et al. (2008).

Finalmente, en el perfil de estudio se han identificado 8 materiales corticales, diferenciándolos en sedimentos (del Terciario, del Mesozoico, del Paleozoico, de la zona Imbricada), corteza superior, corteza inferior y intrusión plutónica. En la Tabla 1 se detallan sus características físicas.

Tabla 1. Parámetros físicos de los diferentes materiales utilizados en la modelización. $\rho$ : densidad $\left[\mathrm{kg} / \mathrm{m}^{3}\right] ; \mathrm{K}$ : conductividad térmica $[\mathrm{W} / \mathrm{K} \cdot \mathrm{m}] ; \mathrm{H}$ : producción de calor radiogénico $\left[\mu \mathrm{W} / \mathrm{m}^{3}\right]$.

\begin{tabular}{|l|c|c|c|}
\hline $\begin{array}{l}\text { Descripción de los } \\
\text { materiales }\end{array}$ & $\boldsymbol{\rho}\left[\mathbf{k g} / \mathbf{m}^{3}\right]$ & $\boldsymbol{K}[\boldsymbol{W} / \mathbf{K} \cdot \boldsymbol{m}]$ & $\boldsymbol{H}\left[\boldsymbol{\mu} \mathbf{W} / \mathbf{m}^{3}\right]$ \\
\hline 1. Sedimentos & 2450 & 2.0 & 1.0 \\
\hline $\begin{array}{l}\text { 2. Sedimentos } \\
\text { Terciarios }\end{array}$ & 2580 & 2.0 & 1.0 \\
\hline $\begin{array}{l}\text { 3. Sedimentos } \\
\text { Mesozoicos }\end{array}$ & 2650 & 2.5 & 1.0 \\
\hline $\begin{array}{l}\text { 4. Sedimentos } \\
\text { Paleozoicos }\end{array}$ & 2700 & 2.5 & 1.0 \\
\hline $\begin{array}{l}\text { 5. Sedimentos Zona } \\
\text { Imbricada }\end{array}$ & 2650 & 2.0 & 1.0 \\
\hline $\begin{array}{l}\text { 6. Granitoide } \\
\text { 7. Corteza superior }\end{array}$ & 2820 & 3.0 & 1.0 \\
\hline 8. Corteza inferior & 2980 & 2.2 & 0.4 \\
\hline
\end{tabular}

\subsection{El manto}

El 98\% del manto terrestre está formado por 5 óxidos principales $(\mathrm{CaO}-\mathrm{FeO}-\mathrm{MgO}-$ $\mathrm{Al}_{2} \mathrm{O}_{3}-\mathrm{SiO}_{2}$ ), el llamado sistema CFMAS, considerado una base excelente para la 
modelización de las fases mantélicas. En nuestro modelo se ha añadido también el óxido de sodio $\left(\mathrm{Na}_{2} \mathrm{O}\right)$. Dada la ausencia de xenolitos en la zona de estudio, se ha usado como fuente de datos de composición mantélica, las ofiolitas del complejo de Sahneh-Kermanshah. Diferentes composiciones (Griffin et al., 2008) de manto han sido empleadas para encontrar el mejor ajuste del modelo.

Considerando que la placa Arábiga es tectónicamente más estable, y que la zona debajo del Zagros es la que está mayormente afectada por la deformación tectónica, desde el punto de vista composicional se ha propuesto un Proton-6 y un Tecton Tc2 (Griffin et al., 2008) para sus respectivos mantos. Para la placa Euroasiática, se ha considerado una composición que procede de datos de las ofiolitas de SahnehKermanshah (Wrobel Daveau et al., 2010). Mantos de composición intermedia se han considerado entre los mantos elegidos y la astenosfera, la cual, composicionalmente corresponde a un PUM (Primitive Upper Mantle) McD \& Sun (Griffin et al., 2008). Las composiciones de los mantos utilizados en la modelización están detalladas en la Tabla 2. La producción de calor en el manto y astenosfera es de 0.02 $\mu \mathrm{W} / \mathrm{m}^{3}$.

Tabla 2. Composición en óxidos principales de los mantos (Griffin et al., 2008; Desmons \& Beccaluva, 1983). A partir de estos datos se calcula la composición mantélica, sus densidades y conductividades.

\begin{tabular}{|l|c|c|c|c|}
\hline \multicolumn{5}{|c|}{ Composiciones Mantos (\%) } \\
\hline $\begin{array}{c}\text { Manto } \\
\text { "Sahneh" }\end{array}$ & Tecton Tc-2 & Proton-6 & $\begin{array}{c}\text { PUM } \\
\text { McD. \& Sun } \\
\text { (astenosfera) }\end{array}$ \\
\hline $\mathbf{S i O}_{2}$ & 38.18 & 45.0 & 45.4 & 45.0 \\
\hline $\mathbf{A l}_{\mathbf{2}} \mathbf{O}_{3}$ & 1.98 & 3.9 & 3.7 & 4.5 \\
\hline $\mathbf{F e O}$ & 8.13 & 8.1 & 8.3 & 8.1 \\
\hline $\mathbf{M g O}$ & 35.67 & 38.7 & 39.9 & 37.8 \\
\hline $\mathbf{C a O}$ & 0.41 & 3.2 & 3.2 & 3.6 \\
\hline $\mathbf{N a}_{2} \mathbf{O}$ & 0.07 & 0.28 & 0.26 & 0.36 \\
\hline
\end{tabular}




\section{Resultados y discusión}

El modelo litosférico que mejor ajusta los observables, anomalía de Bouguer, geoide y elevación, está ilustrado en la Figura 4. No se tienen datos de flujo de calor a lo largo del perfil (Fig. 4 a). Aunque las figuras se muestran solo hasta una profundidad de $250 \mathrm{~km}$, el modelo ajusta las variables hasta $400 \mathrm{~km}$ de profundidad.

En línea general el modelo ajusta bastante bien los datos, aunque hay algunas zonas en las cuales efectos locales causan un ligero desajuste.

El adelgazamiento de la litosfera debajo del Zagros es la característica más remarcable de este modelo. El espesor litosférico aumenta debajo de la cuenca Mesopotámica hasta $\sim 230 \mathrm{~km}$ de profundidad, para luego disminuir hacia $\sim 120 \mathrm{~km}$ debajo de la Sinandaj Sirjan Zone. El adelgazamiento se extiende por debajo del Zagros y del Alborz, aunque haya un leve engrosamiento en el sector del arco magmático Urumieh Dokhtar correspondiente a una topografía más suave entre las dos cadenas montañosas.

Debajo del Zagros tenemos material caliente astenosférico que asciende más de $100 \mathrm{~km}$, calentando el manto supra yacente y por lo tanto modificando los equilibrios de fases de los minerales del manto. En la distribución de temperatura (Fig. 5) el cambio en las isotermas es notable. La temperatura en la Moho varía entre $530^{\circ} \mathrm{C}$ en la cuenca Mesopotámica a $800^{\circ} \mathrm{C}$ y $750^{\circ} \mathrm{C}$ debajo del Zagros y del Alborz, respectivamente. El límite litosfera-astenosfera está definido a $1330^{\circ} \mathrm{C}$.

En relación a las densidades mantélicas (Fig. 6), los valores varían de 3320 $\mathrm{kg} / \mathrm{m}^{3}$ en la base de la corteza, a los $3450 \mathrm{~kg} / \mathrm{m}^{3}$ a $250 \mathrm{~km}$ de profundidad. Se observa una zona de menor densidad debajo del orógeno con valores entre 3320-3340 $\mathrm{kg} / \mathrm{m}^{3}$, relacionada con el aumento de temperatura observado en la misma zona. Las variaciones de densidad en el manto litosférico son debidas a las variaciones composicionales. a la temperatura y a la presión.

Observando las velocidades de las ondas sísmicas obtenidas en el manto (Fig. 7), se encuentran valores que concuerdan con los de estudios tomográficos en la zona (Maggi and Priesley, 2005; Kaviani et al., 2007). Los valores están comprendidos entre $8.0 \mathrm{~km} / \mathrm{s}$ y $8.4 \mathrm{~km} / \mathrm{s}$ para las ondas $P$ y entre $4.41 \mathrm{~km} / \mathrm{s}$ y $4.7 \mathrm{~km} / \mathrm{s}$ para las ondas $\mathrm{S}$. 


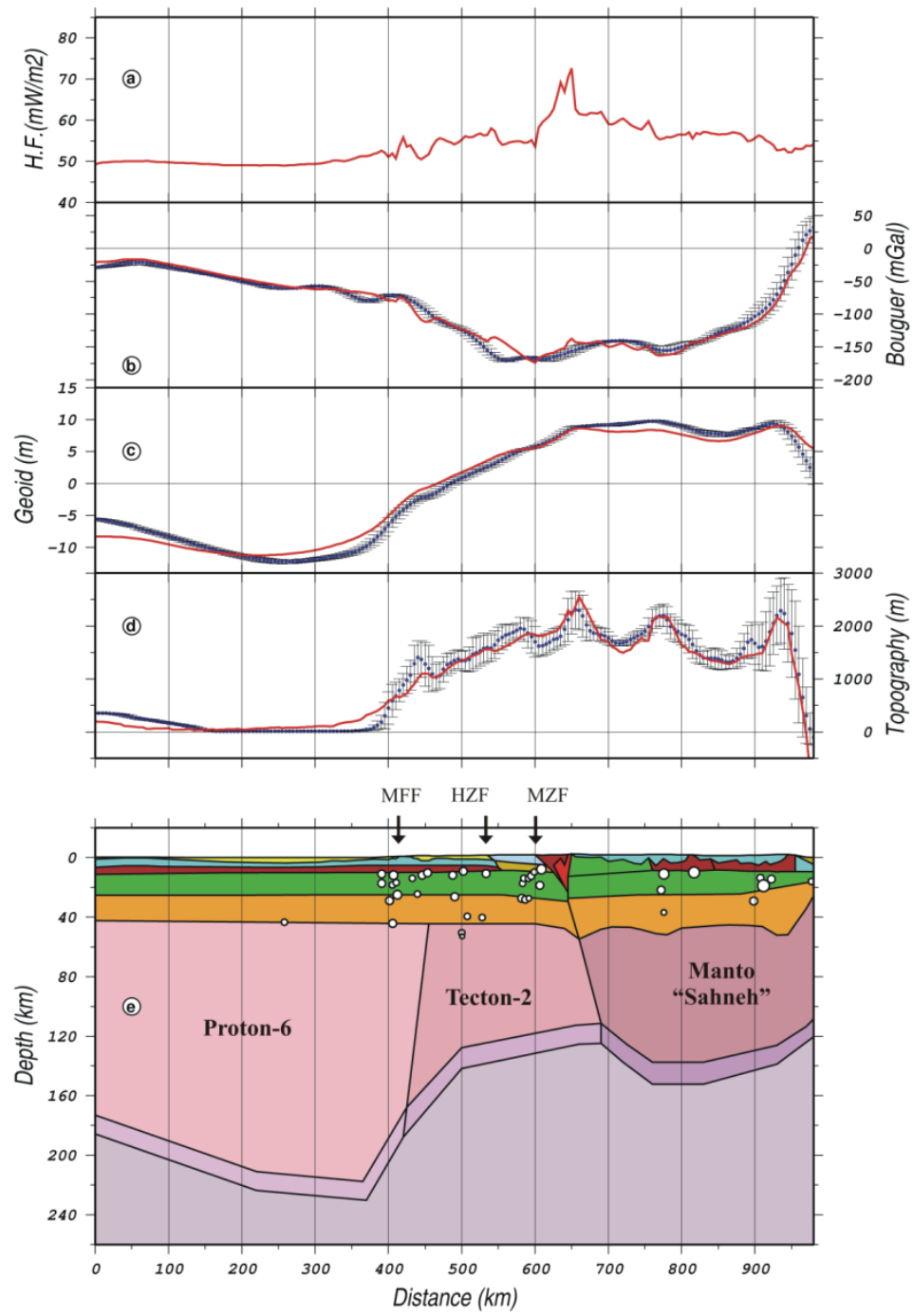

Fig. 4. Resultado de la modelización del perfil (líneas rojas). Los datos son los puntos azules y las barras verticales corresponden a la desviación estándar dentro de una banda de $25 \mathrm{~km}$ por cada lado del perfil. a). Flujo de calor $\left(\mathrm{mW} / \mathrm{m}^{2}\right)$. Se ha recopilado datos de flujo de calor en la base de datos de la International Heat Flow Commission (actualizados en enero 2011) pero no hay datos en la zona de estudio (en un rango de $200 \mathrm{~km}$ para cada lado del perfil). b) Anomalía de Bouguer (mGal); c) Altura del geoide (m); d) Elevación (m); e) Estructura de la corteza y de la litosfera. Las líneas oblicuas en el manto representan los límites de las diferentes composiciones mantélicas detalladas en la Tabla 2. Los círculos representan los hipocentros de los terremotos principales (catálogo Engdahl). 


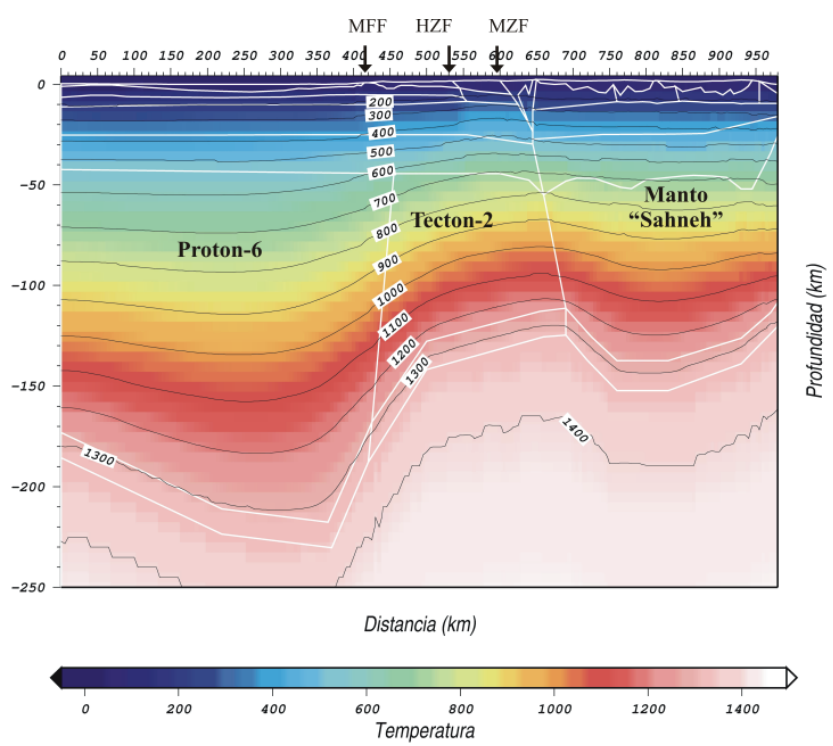

Fig. 5. Distribución de la temperatura $\left({ }^{\circ} \mathrm{C}\right)$ en el manto litosférico a lo largo del perfil estudiado.

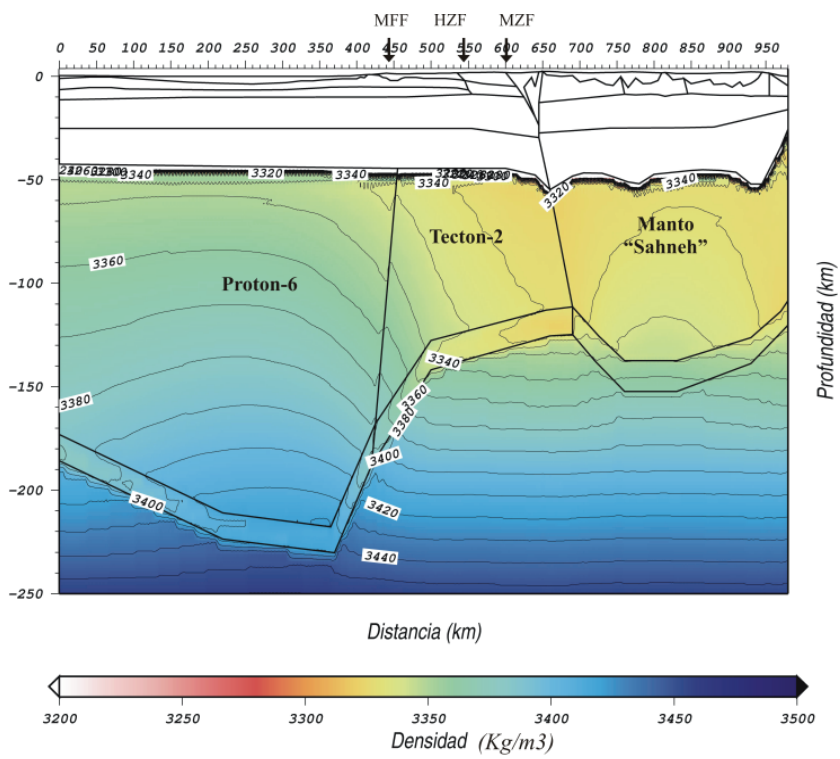

Fig. 6. Distribución de densidad $\left(\mathrm{kg} / \mathrm{m}^{3}\right)$ en el manto litosférico, a lo largo del perfil estudiado. 

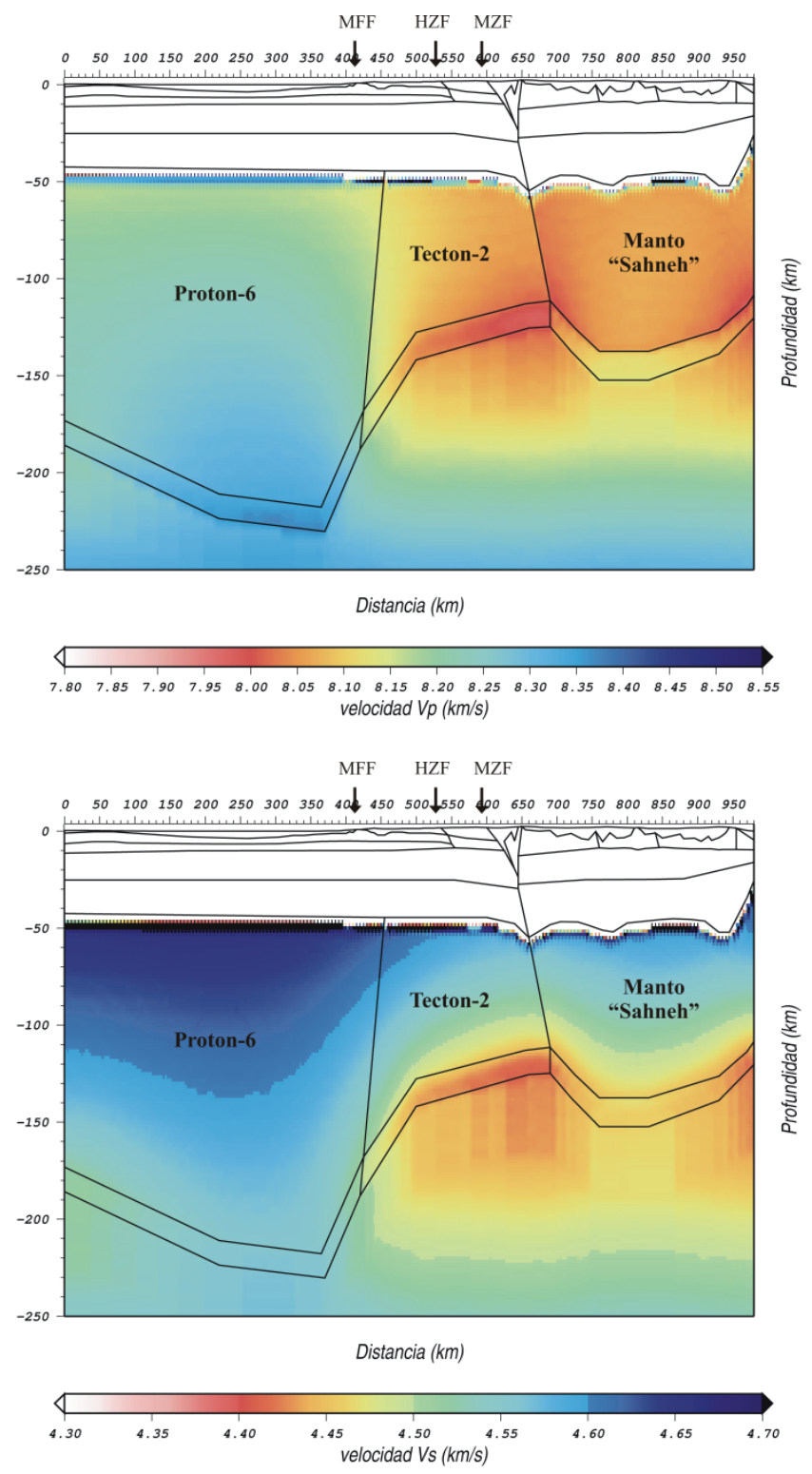

Fig. 7. Distribuciones de las velocidades sísmicas $(\mathrm{km} / \mathrm{s})$ de las ondas $\mathrm{P}$ (arriba) y S (abajo) a lo largo del perfil estudiado. 


\section{Conclusiones}

En este trabajo se ha investigado la estructura profunda (hasta una profundidad de $400 \mathrm{~km}$ ) a lo largo de un perfil que corta perpendicularmente el Zagros, empezando en la cuenca Mesopotámica y llegando a la costa Sur del Mar Caspio, cruzando la Zagros Folded Thrust Belt, la Zona Imbricada, la Sinandaj Sirjan Zone y las montañas del Alborz. Gracias a la combinación de las variables geofísicas empleadas (geoide, anomalía de Bouguer, flujo térmico, elevación), y constriñendo el modelo con datos de geología y petrografía, conseguimos determinar la base de la corteza, el LAB y la composición mantélica.

Durante la modelización, resultó evidente que no era posible modelar la elevada topografía del Zagros solo a través de un engrosamiento cortical debido a la convergencia de placas, sino que había que considerar una menor densidad en el manto para justificar su elevación, anomalías del geoide y gravimetría. En el modelo presentado, la expresión de esta anomalía negativa de densidad es un adelgazamiento del espesor litosférico. Este déficit de masa comprende toda la zona debajo del Zagros y del Alborz, y está acompañado por una temperatura más elevada. La base de la litosfera, que debajo del Foreland Basin roza los $230 \mathrm{~km}$ de profundidad, se levanta hacia los $\sim 120 \mathrm{~km}$ debajo de la Sinandaj Sirjan Zone. El modelo ajusta bien los datos si se consideran tres composiciones diferentes: un Proton-6 (Griffin et al., 2008) para el manto debajo de la cuenca Mesopotámica; un Tecton Tc-2 (Griffin et al., 2008) para el manto adelgazado debajo del orógeno, y una composición diferente, procedente de la composición de las ofiolitas de Sahneh, para el manto debajo de la placa Eurasiática.

El modelo presentado ajusta todas las variables geofísicas introducidas, determina las composiciones mantélicas y calcula velocidades de ondas sísmicas. Sin embargo este resultado representa solo un primer paso en el estudio de la estructura profunda del Zagros. En el próximo futuro se realizaran análisis de las velocidades sísmicas obtenidas comparándolas con las observadas en tomografías sísmicas, de ondas Pn y ondas superficiales, para obtener modelos aún más realistas. También se investigará la estructura profunda a lo largo de otro perfil, paralelo y situado más al sur, para seguir el variación de la estructura a lo largo de toda la cadena del Zagros.

\section{Agradecimientos}

Este trabajo ha sido financiado por el Proyecto ATIZA (CGL2009-09662-BTE) y TopoMed/GASAM (CGL2008-03474-E/BTE/07-TOPO-EUROPE-FP-006)). Se agradece a J. Vergés, J.C. Afonso y J. Fullea por las interesantes discusiones mantenidas.

\section{Referencias bibliográficas}

AFONSO C., FERNÁNDEZ M., RANALLI G., GRIFFIN W.L., CONNOLLY J.A.D. (2008). Integrated geophysical-petrological modeling of the lithosphere and sublithospheric upper mantle: methodology and applications. Geochem. Geophys. Geosyst.,vol. 9, Q05008, http://dx.doi.org/10.1029/2007GC001834 
AGARD, P., OMRANI J., JOLIVET J., MOUTHEREAU, F. (2005). Convergence history across Zagros (Iran): constraints from collisional and earlier deformation. Int. J. Earth Sc. (Geol Rundsch), v. 94, p. 401-419, http://dx.doi.org/10.1007/s00531-005-0481-4

ALAVI, M. (1994). Tectonics of the Zagros orogenic belt of Iran: new data and interpretations. Tectonophysics, v. 229, p. 211-238. http://dx.doi.org/10.1016/00401951(94)90030-2

ALINAGHI, A., KOLAKOV I., THYBO H. (2007). Seismic tomographic imaging of P- and S-waves velocity perturbations in the upper mantle beneath Iran. Geoph. J. Int., 169, 1089-1102, http://dx.doi.org/10.1111/j.1365-246X.2007.03317.x

BERBERIAN, M. G.C.P. KING, (1981) Towards a palegeography and tectonic evolution of Iran, Can. J. Earth Sc., 18, 210-265. http://dx.doi.org/10.1139/e81-019

CASCIELlO E., VERGES J., SAURA E., CASINI G., FERNANDEZ N., BLANC E., HOMKE S., HUNT, D. W. (2009). Fold patterns and multilayer rheology of the Lurestan Province, Zagros simply folded belt (Iran). J. of the Geological Society of London, 166 (5), 947-959. http://dx.doi.org/10.1144/0016-76492008-138

DEHGHANI, G.A., MAKRIS J. (1984). The gravity field and crustal structure of Iran, Neues Jahrbuch fur Geologie und Palaeontologie, Abhandlungen, 168, 215-229.

DESMONS J., BECCALUVA L. (1983). Mid-ocean ridge and island-arc affinities in ophiolites from Iran: paleographic implications. Chemical Geology, 39, 39-63. HTTP://DX.DOI.ORG/10.1016/0009-2541(83)90071-2

ENGDAHL, E. R., VAN DER HILST R. D., BULAND R. P. (1998). Global teleseismic earthquake relocation with improved travel times and procedures for depth determination. Bulletin of the Seismological Society of America, 88 (3):722-743

FULLEA, J., FERNANDEZ M., AND ZEYEN H. (2008). FA2BOUG - A FORTRAN 90 code to compute Bouguer gravity anomalies from gridded free-air anomalies: Application to the Atlantic-Mediterranean transition zone. Computers \& Geosciences, 34, 1665-1681, HTTP://DX.DOI.ORG/10.1016/J.CAGEO.2008.02.018

GETECH, HTTP://WWW.GETECH.COM/

GHASEMI A. TALBOT C.J. (2006). A new tectonic scenario for the Sanandaj-Sirjan Zone (Iran). Journal of Asian Earth Sciences, 26, 683-693 http://dx.doi.org/10.1016/j.jseaes.2005.01.003

GRIFFIN, W.L., O'REILLY S.Y., AFONSO J.C., BEGG G., (2008). The composition and evolution of lithospheric mantle: a re-evaluation and its tectonic implications. J. Petrol. http://dx.doi.org/10.1093/petrology/egn033

HATZFELD, D., TATAR M., PRIESTLEY K., GHAFORY-ASHTIANY M. (2003). Seismological constraints on the crustal structure beneath the Zagros Mountain belt (Iran). Geophys. J. Int., 155, 403- 410.k HTTP:/DX.DOI.ORG/10.1046/J.1365246X.2003.02045.X

HOMKE S., VERGES J., SERRA-KIEL J., BERNAOLA G., SHARP I., GARCES M., MONTERO-VERDU I., KARPUZ R., HASSAN GOODARZI M. (2009). Late Cretaceous-Paleocene formation of the proto-Zagros foreland basin, Lurestan Province, SW Iran. Geological Society of America Bulletin, 121 (7-8), 963-978. http://dx.doi.org/10.1130/B26035.1 
KAVIANI, A., PAUl A., BOUROVA E., HATZFELD D., PEDERSEN H., MOKHTARI M. (2007). A strong seismic velocity contrast in the shallow mantle across the Zagros collision zone (Iran). Geophys. J. Int., 171: 399-410, http://dx.doi.org/10.1111/j.1365246X.2007.03535.X

MAGGI A., PRIESTLEY K. (2005). Surface waveform tomography of the Turkish- Iranian plateau. Geoph. J. Int., 160, 1068-1080. http://dx.doi.org/10.1111/j.1365246X.2005.02505.x

MOLINARO M., ZEYEN H., LAURENCIN X., (2005). Lithospheric structure beneath the southeastern Zagros Mountains, Iran: recent slab break-off?. Terra Nova, 17, 1- 6, http://dx.doi.org/10.1111/j.1365-3121.2004.00575.x

MOTAVALLI ANBARAN, S.H., ZEYEN H. J., BRUNET M., ARDESTANI V. E. (2010). Crustal and lithospheric structure of the Alborz Mountains (Iran) and surrounding areas from integrated geophysical modeling. Eos Trans. AGU, 91 (52), Fall Meet. Suppl., Abstract T43C-2249, AGU, San Francisco, USA, 13-17 Dec. 2010.

NASRABADI A., TATAR M., PRIESTLEY K., SEPAHVAND M. R. (2008). Continental lithosphere structure beneath the Iranian plateau, from analysis of receiver functions and surface waves dispersion. 14th World Conference on Earthquake Engineering, October 12-17, 2008, Beijing, China.

PAUL, A., HATZFELD D., KAVIANI A., TATAR M., PÉQUEGNAT C. (2010). Seismic imaging of the lithospheric structure of the Zagros mountain belt (Iran). Geol. Society of $\begin{array}{lrll}\text { LONDON, SPECIAL } & \text { PUBLICATIONS, } & 330,\end{array}$ HTTP://DX.DOI.ORG/10.1144/SP330.2

PAVLIS N.K., HOLMES S.A., KENYON S.C., FACTOR J.K. (2008). An Earth Gravitational Model to Degree 2160: EGM 2008. GRACE Science Applications, session G3, EGU 2008, Vienna.

RADJAEE, A., RHAM D., MOKHTARI M., TATAR M., PRIESTLEY K., HATZFELD D. (2010). Variation of Moho depth in the central part of the Alborz Mountains, northern Iran. Geophys. J. Int., 181, 173-184. http://dx.doi.org/10.1111/j.1365246X.2010.04518.x

RICOU, L.E. (1971). Le croissant ophiolitique peri-arabe. Une ceinture de nappes mises en place au Cretace superieur. Rev. Geogr. Phys. Geol. Dyn., 13, 327-350.

SANDWELL, D. T., SMITH H. W. F. (1997). Marine gravity anomalies from GEOSAT and ERS-1 satellite altimetry. J. Geophys. Res., 102, 10,039 - 10,054. HTTP://DX.DOI.ORG/10.1029/96JB03223

SNYDER D. B., BARAZANGI M. (1986). Deep crustal structure and flexure of the Arabian plate beneath the Zagros collisional mountain belt as inferred from gravity $\begin{array}{llll}\text { OBSERVATIONS. TECTONICS, } & \text { 361-373. }\end{array}$ HTTP:/DX.DOI.ORG/10.1029/TC005I003P00361

SODOUDI, F., YUAN X., KIND R., HEIT B., SADIDKHOUY A. (2009). Evidence for a missing crustal root and a thin lithosphere beneath the Central Alborz by receiver function studies, Geophys. J. Int., 177, 733-742. http://dx.doi.org/10.1111/j.1365246X.2009.04115.X

VERGÉS J., SAURA E., CASCIELlO E., FERNÁNDEZ M., VILLASEÑOR A., JIMÉNEZ-MUNT I., GARCÍA-CASTELLANOS D. (2011). Crustal-scale cross- 
sections across the NW Zagros belt: implications for the Arabian margin reconstruction. Geological Magazine, http://dx.doi.org/10.1017/S0016756811000331

WROBEL-DAVEAU, J.C., RINGENBACH J.C., TAVAKOLI S., RUIZ G.M.H., MASSE P., LAMOTTE D.F. (2010). Evidence for mantle exhumation along the Arabian margin in the Zagros (Kermanshah area, Iran). Arabian Journal of Geosciences. http://dx.doi.org/10.1007/s12517-010-0209-z.

ZIEGLER, M.A. (2001). Late Permian to Holocene paleofacies evolution of the Arabian plate and its hydrocarbon occurrences. Geoarabia, 6, 445-504. 